\title{
Sleep disorders - another perspective
}

\begin{abstract}
Sleep disorders are usually multi-factorial in nature, very often without a single solution available. As with many interventive processes, there are unanticipated iatrogenic consequences, which cause further complications, and more intervention is then required. Sufficient evidence now exists to indicate that prevention of the onset of the symptoms, which are often referred to as 'the disease', can minimize or even eliminate the need for intervention. It is not possible for a person to have functional breathing during the day and dysfunctional breathing during the night, any more than it is to have the reverse situation. Given that the vast majority of people spend approximately two thirds of their lives awake and active, there is a significant likelihood that addressing daytime dysfunction is at least as important as, if not more important than, applying short - term nighttime intervention.
\end{abstract}

Volume 2 Issue 4 - 2015

\section{Roger L Price}

Respiratory Physiologist and Integrative Health Educator, USA

Correspondence: Roger L Price, Respiratory Physiologist and Integrative Health Educator, Breathing well LLC, 1425 Broad St Ste A, Clifton NJ 070 I3, USA, Tel (973)5 I0-0432, (505)33 I-I05I, Email rogeriprice@gmail.com, breathingwellopt.com

Received: March 06, 2015 | Published: April 15, 2015

\section{Introduction and commentary}

In this era of 'spin', selective reporting of fact, and downright untruths, it is difficult to know exactly what or who to believe. The fact is that the so - called "Health System" really has very little to do with health, but is rather a professionally managed, obscenely profitable business, based on "Disease Creation and Management". It is sad that human nature is such that when an opportunity is created to 'scam' or 'game' a system, there is no shortage of opportunists ready to cash in on ignorance, fear or distress. When we see regular examples of how the true results of clinical trials have been manipulated, selectively reported, and sometimes even totally obscured, in order to present a favorable commercial outcome, ${ }^{2}$ it is no wonder that a healthy degree of cynicism prevails in the sector of the profession that is more concerned with Outcome than Income. Sleep Disorders are usually complex mixtures of various compromises, compensations, para functions and dysfunctions-all ending up in a situation where health is compromised through lack of quality sleep. Given this complexity in etiology, it is unlikely that the offer of quick solutions has much validity.

Similarly, the current rash of offers of becoming an "expert in sleep dentistry" after an expensive 2 day weekend, have to be looked at askance - and closer examination of some of the processes involved, show a distinct bias towards exploiting loopholes in the legislation and insurance billing, ${ }^{3}$ where the focus is on fitting as many oral appliances as possible - irrespective of whether they are appropriate, or actually address the sleep disorder effectively. So based on the above, one does not need a long stretch of the imagination to see how the following could well apply to many of the 'truths' that are currently being paraded as the answer to all Sleep Disorders and other comorbid conditions.

"tell a lie, loudly and often enough, and it becomes the truth" attributed to both Lenin and Goebbels "if all you have is a hammer, everything looks like a nail" Abraham Maslow's The Psychology of Science, 1966

\section{Generally accepted standards and guidelines}

So let us look at the Gold Standards, Best Practice and Evidence Based practices currently in use today. The industry is rife with many
TLAs (three letter acronyms), the majority of which have ZERO empirical value, and, at best, are nothing more than useful indicators of change. Again however, the fact that a 'number' exists, even if, like the BMI, it bears NO resemblance to the individual - but is rather an indicator of a group tendency - it is still used as an empirical diagnostic icon in the determination of a condition. Similarly, names given to conditions, or groups of symptoms, take on a life of their own, and, through repetition and reinforcement, become the 'buzzwords' of the industry - without actually meaning much. Furthermore, there is the temptation to 'grab - bag' symptoms into neat and tidy definitions, which, through usage, become non - defining definitions. An example here is the use of the word 'flu to describe everything from a minor sore throat, or a mild cold virus-right up to life threatening influenza. Let us examine the term SDB- Sleep Disordered Breathing - used as a grab - bag for anything to do with sleep and breathing. What does it actually mean? What is Sleep - Disordered - Breathing?

\section{American Thoracic Society Definition}

Sleep-disordered breathing is an umbrella term for several chronic conditions in which partial or complete cessation of breathing occurs many times throughout the night, resulting in daytime sleepiness or fatigue that interferes with a person's ability to function and reduces quality of life.

\section{Medicine.net}

Sleep-disordered breathing: A condition characterized by repeated episodes of hypopnea (under breathing) and apnea (not breathing) during sleep. A significant proportion of adults are thought to experience sleep-disordered breathing.

\section{Cleveland Clinic}

SDB comprises a wide spectrum of sleep-related breathing abnormalities; those related to increase upper airway resistance include snoring, upper airway resistance syndrome (UARS), and obstructive sleep apnea-hypopnea syndrome (OSAHS). Many clinicians regard $\mathrm{SDB}$ as a spectrum of diseases.

\section{Resmed}

Sleep Disordered Breathing (SDB) describes a group of disorders characterized by: 
i. Abnormal respiratory patterns (e.g. the presence of apneas or hypopneas);

ii. Insufficient ventilation during sleep

There doesn't seem to be a sufficient commonality in the above definitions to be able to zero in on what causes the actual problem - so the only option available is to use short-term intervention to attempt to manage the symptoms when they occur-usually during the night. If, on the other hand, one was to look at the non-sleep behavior - the 16-18 hours a day when the patient is awake, working, driving, eating etc. does it not make sense to examine this behavior to see if what they are doing - for three times the amount of time that they are in bed - can influence their condition to a greater extent than the few hours they're trying to sleep?

This is why there is so much validity in the concept of Breathing Disordered Sleep (BDS) as the etiology of the problem - rather than the other way round. There is no doubt that there are millions of people suffering from Sleep Disorders, and that only a very small percentage is being reported-and successfully treated - and it is beyond important that we try to understand why this is so. This is a multi-billion dollar industry which, by its own reporting, is perhaps addressing less than $10 \%$ of the suspected cases of Sleep Disorders. One doesn't have to be a genius to see the disproportionate benefit, or lack thereof, in such vast amounts of money being spent on so small a segment of the market. What is of even greater concern is the abysmally low compliance rate of these devices-and the actual cost per successful patient outcome-is staggering.

Breathing is a mainstream function - Why then Should Breathing Retraining be regarded as Complementary or Alternative?

\section{Breathing disordered sleep}

\section{Cause and effect}

Where better to find the etiology than in the simple Newtonion Third Law of Motion?

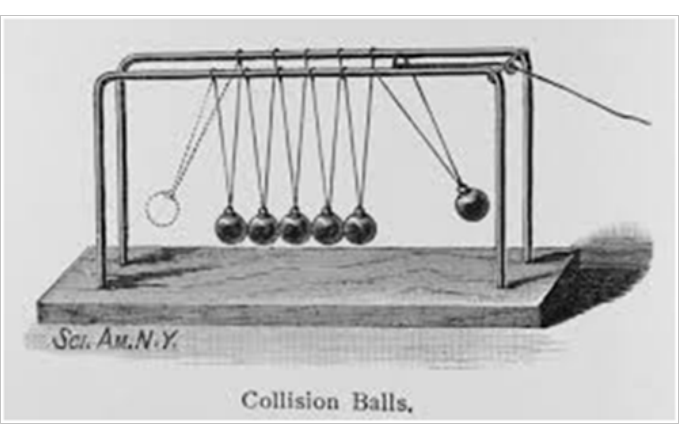

Because "every action has an equal and opposite reaction" it is not a long bow to draw between linking daytime dysfunction to nighttime consequences. The perennial complaint in the sleep industry is lack of compliance. So serious is this issue that the term 'compliance' has been watered down to mean far less than its actual description. The Medicare definition of CPAP compliance is "equal to or greater than 4 hours per night for $70 \%$ of nights in a consecutive 30 day period."

So the question arises. "Why is compliance so poor?" It is hard to believe that people with a serious sleep disorder would not want something to alleviate their condition - so why is it so difficult to get them to comply? The answer could be much simpler than is widely believed - and the explanation that follows might shed some light on this perplexing problem.

\section{Explanation}

Breathing is triggered by a sensor in the medulla portion of the brainstem that responds to both $\mathrm{pH}$ shift and the arterial pressure of $\mathrm{CO}_{2}$. The majority of textbooks on this subject admit that the mechanism is not fully understood, but it is widely accepted that an increase in $\mathrm{CO}_{2}$ levels is what stimulates breathing. Does it not then stand to reason that if the brainstem becomes hyper-responsive to the $\mathrm{pH} / \mathrm{CO}_{2}$ trigger, that breathing will be initiated earlier than 'normal'?

Hyperventilation - http://www.nlm.nih.gov/medlineplus/ency/ article/003071.htm

Hyperventilation is rapid or deep breathing that can occur with anxiety or panic. It is also called over breathing, and it may leave you feeling breathless. You breathe in oxygen and breathe out carbon dioxide. Excessive breathing creates low levels of carbon dioxide in your blood. This causes many of the symptoms of hyperventilation.

Given that Hyperventilation is an accepted medical diagnosis, can become a serious chronic condition, and can lead to the re-setting of the brainstem response, is it not possible that a sudden reduction in breathing rate-such as caused by CPAP or some Oral Appliances, could result in a spike in $\mathrm{PaCO}_{2}$ ? This would then cause the breathing rate to rise-which is not permitted by the machine and mask-and be one of the reasons that patients experience a 'panic, claustrophobic or drowning' feeling, and have to remove the intervention? This mechanism will be discussed in greater detail later. Absent the fact that there is no such thing as normal breathing - the best we can rely on is a scientifically calculated formula for 'optimal breathing at rest'. Obviously, breathing changes all the time, to address function and effort, but measurement at rest gives us a fairly accurate baseline from which to evaluate functional efficiency.

\section{Breathing is a mainstream function - Why then should Breathing retraining be regarded as Complementary or Alternative?}

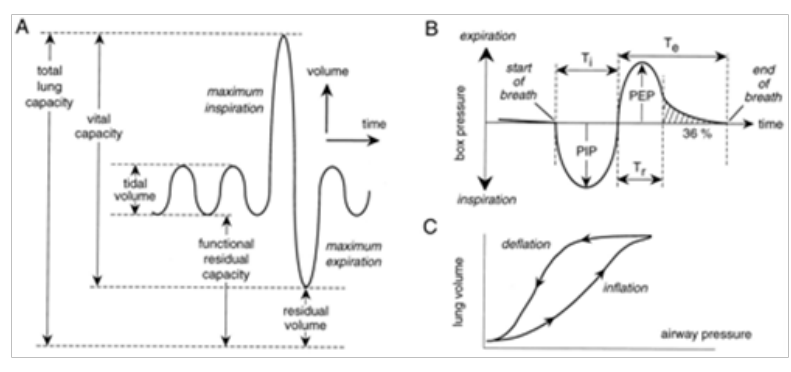

\section{Terminology}

i. Minute Volume (MV), The volume of air inhaled and exhaled in one minute.

ii. Respiratory Rate (RR) (also referred to as breaths per minute), The number of inhalation/exhalation cycles in one minute.

iii. Tidal volume (TV) about $500 \mathrm{~mL}$, is the amount of air inspired during normal, relaxed breathing.

iv. Inspiratory reserve volume (IRV), about $3,100 \mathrm{~mL}$, is the additional air that can be forcibly inhaled after the inspiration of a normal tidal volume.

v. Expiratory reserve volume (ERV), about $1,200 \mathrm{~mL}$, is the additional air that can be forcibly exhaled after the expiration of a normal tidal volume. 
vi. Residual volume (RV), about $1,200 \mathrm{~mL}$, is the volume of air still remaining in the lungs after the expiratory reserve volume is exhaled.

vii. Summing specific lung volumes produces the following lung capacities: Total lung capacity (TLC), about $6,000 \mathrm{~mL}$, is the maximum amount of air that can fill the lungs $(\mathrm{TLC}=\mathrm{TV}+\mathrm{IR}-$ $\mathrm{V}+\mathrm{ERV}+\mathrm{RV})$.

viii. Vital capacity (VC), about $4,800 \mathrm{~mL}$, is the total amount of air that can be expired after fully inhaling $(\mathrm{VC}=\mathrm{TV}+\mathrm{IRV}+\mathrm{ER}-$ $\mathrm{V}=$ approximately 80 percent TLC). The value varies according to age and body size.

ix. Inspiratory capacity (IC), about $3,600 \mathrm{~mL}$, is the maximum amount of air that can be inspired ( $\mathrm{IC}=\mathrm{TV}+\mathrm{IRV})$

x. Functional residual capacity (FRC), about $2,400 \mathrm{~mL}$, is the amount of air remaining in the lungs after a normal expiration $(\mathrm{FRC}=\mathrm{RV}+\mathrm{ERV})$.

Some of the air in the lungs does not participate in gas exchange. This air is located in the anatomical dead space within bronchi and bronchioles - that is, outside the alveoli. By using the above information we can arrive at what constitutes functional breathing at rest - and that can be summarized in a few lines.

i. $8-10$ breaths per minute

ii. 4- 5 liters of air per minute

iii. In and out through the nose

iv. Driven by the diaphragm - not upper chest

v. Silent

When a person breathes at this rate the $\mathrm{ETCO}_{2}$ (end tidal $\mathrm{CO}_{2}$ ) reaches approximately $40 \mathrm{~mm} \mathrm{Hg}$ pressure, there is sufficient buffer available for the $\mathrm{pH}$ in the respiratory system to move between 7.35 and 7.45, oxygen transport and release is optimal, and smooth muscle

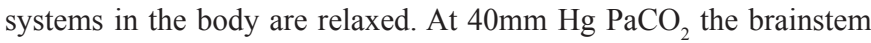
response maintains this rhythm. What then happens when people breathe dysfunctionally? They breathe more air per breath, more breaths per minute, start to use mouth and chest instead of nose and diaphragm, and reduce their $\mathrm{ETCO}_{2}$. This alters the respiratory $\mathrm{pH}$ resulting in a tendency towards more alkalinity, and as a consequence, the release of oxygen from the hemoglobin is reduced. (The Bohr Effect). Concurrently, as a compensatory defense mechanism against further loss of $\mathrm{CO}_{2}$, broncho constriction occurs - prompting even more labored breathing. Should the reduction in $\mathrm{CO}_{2}$ loss not be sufficient, the next line of defense is a breath-hold or apnea - to rapidly increase retained $\mathrm{ETCO}_{2}$ and therefore $\mathrm{PaCO}_{2}$.

This is done to ensure the ongoing supply of oxygen to the brain and the rest of the body. Ironically - an apnea is nature's way of protecting the body - not harming it. Many doctors totally refute the idea that it is possible to reprogram the brainstem response - and demand scientific proof to support this assertion. They furthermore claim that breathing is a totally automatic response and, as such, cannot be changed. If it is not possible to change the brainstem response why then do babies, born as obligate nasal breathers, develop into mouth breathers in a short space of time?

Why is it that functional minute volume, long held to be around 6 liters per minute, is now regarded as being 'normal' at 12 liters per minute?

If this is not indicative of a change in the brainstem breathing response - what is?

The reality is that the brainstem response IS subject to reprogramming - just as much as so many other body functions alter in the face of long term dysfunctional behavior. So - in summary Breathing Disordered Sleep is based on the following premises:

i. Long term stress and dysfunction causes hyperventilation.

ii. Hyperventilation increases the brainstem response to $\mathrm{CO}_{2}$ and is a known precursor to many sleep disorders - especially Central Sleep Apnea.

iii. Rapidly moving someone from hypocapnia to normocapnia or even hypercapnia, provokes an instantaneous response and an automatic reaction to remove the source of interference.

iv. By addressing daytime dysfunction and parafunctions, and by retraining the brainstem response to be less reactive to $\mathrm{CO}_{2}$ it is possible to reduce the onslaught-effect of CPAP and/or OAT, make them easier to adapt to, and therefore achieve better compliance with less stress and emotional trauma.

v. Simple common sense should show that it is more likely that 16-18hours of dysfunctional behavior during the dayhas the potential to influence night time breathing more than 6-8hours of night time dysfunction has the potential to disrupt daytime balance.

vi. It is not possible for breathing to be functional during the day and not at night, any more than being functional at night and not during the day.

\section{The iceberg analogy}

The $10 \%$ of the iceberg that is visible is not what presents the danger. It is the $90 \%$ hidden below the surface that sinks ships. This diagram indicates the ratio of symptoms to etiology - and if one takes into consideration the billions of dollars expended on above-the-line intervention, against the almost negligible focus on the below-theline causes or exacerbatory factors, it is no wonder that the problems remain unresolved to the extent that they are.

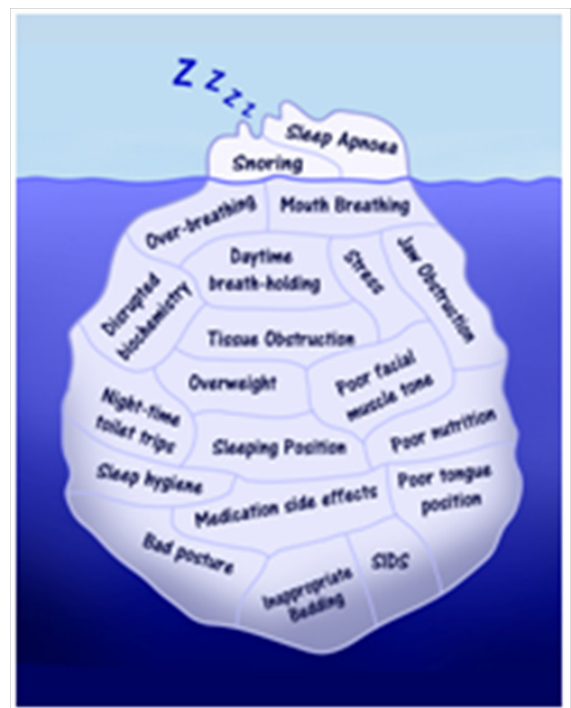


Therefore addressing the 'below-the-line' issues such as posture, breathing mechanics, nutrition, stress and the rest of the co-factors would go a long way in providing a more comfortable, acceptable and stable outcome - with far greater compliance.

Ignoring the presence of these multiple factors will result in being confined to short-term night-time intervention. Handling such multi-factorial issues requires the involvement of a trained team of therapists, working in conjunction, to get the required result.

\section{Conclusion}

Just because something is being done by a lot of people don't necessarily mean that this is the best or most successful way of doing things. Change is challenging - especially when there is pressure from commercial interests to maintain their bottom line by insisting on selling equipment and appliances - even when common sense indicates that there are better alternatives. So, based on the research papers attached - which show conclusively that restoring normocapnia will reverse CSA - does it not make sense to change breathing behavior to prevent the loss of the $\mathrm{CO}_{2}$ in the first place - through over breathing?

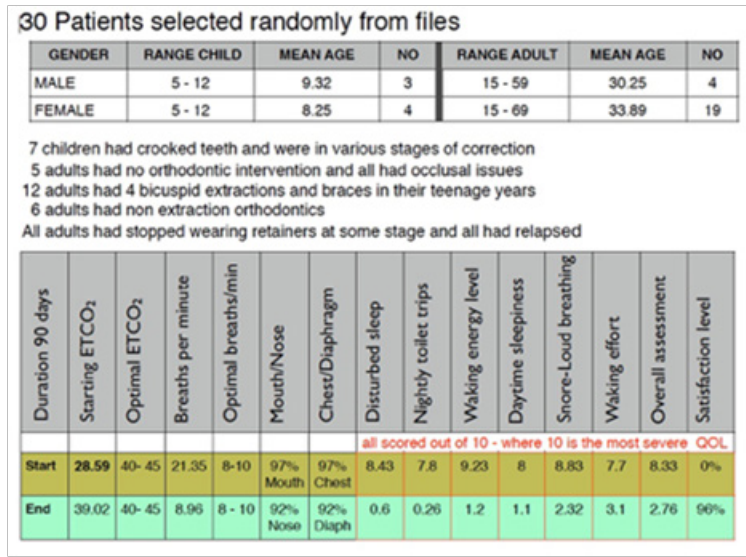

"The pathophysiology is a combination of factors which involve the brain, the heart and lungs. A key factor is enhanced chemoreceptor responses-the degree to which you hyperventilate, in response to hypoxia and hypercapnia.

A characteristic feature of CSA is hyperventilation, which is where it has its parallels with altitude sickness. The difference is that in altitude sickness it's hypoxia that is the primary factor driving hyperventilation and hypocapnia is a consequence of that. In heart failure there's increased ventilation due to a number of factors including the fact that the lungs are wet and receptors in the lungs are sending nerve signals to the respiratory control centers in the brainstem to cause hyperventilation. In healthy individuals carbon dioxide levels normally rise during sleep. If we go to sleep with a low $\mathrm{CO}_{2}$ level below a certain value (the apnea threshold') breathing will be inhibited and reduce or even stop until the $\mathrm{CO}_{2}$ rises, when breathing resumes.

It is common in patients with heart failure, and healthy individuals at altitude, for hyperventilation and the resultant hypocapnia to make breathing unstable during sleep. In patients with heart failure and sleep-disordered breathing, breathing is made more unstable as the prolongation of circulation time means that the effects of blood gas changes in the periphery take longer to reach the brainstem where control of breathing occurs.

\section{Acknowledgements}

None.

\section{Conflict of interest}

The author declares no conflict of interest.

\section{References}

I. Xie A, Rankin F, Ruther ford R, et al. Effects of inhaled $\mathrm{CO}_{2}$ and added dead space on idiopathic central sleep apnea. J Appl Physiol. 1985;82(3):918-926.

2. Badr MS, Grossman JE, Weber SA. Treatment of refractory sleep apnea with supplemental carbon dioxide. Am J Respir Crit Care Med. 1994; I50(2):56I-564.

3. Thomas RJ, Daly RW,Weiss JW. Low-concentration carbon dioxide is an effective adjunct to positive airway pressure in the treatment of refractory mixed central and obstructive sleep-disordered breathing. Sleep. 2005;28(I):69-77.

4. Szollosi I, Jones M, Morrell MJ, et al. Effect of $\mathrm{CO}_{2}$ inhalation on central sleep apnea and arousals from sleep. Respiration. 2004;7I(5): 493-498.

5. Johansen T, Jack S, Dahl R. Normalizing $\mathrm{CO}_{2}$ in chronic hyperventilation by means of a novel breathing mask: a pilot study. Clin Respir J. 2013;7(4):359-366.

6. Central Sleep Apnea Treatment \& Management.

7. Ritz T, Rosenfield D, Steele AM, Millard MW, Meuret AE (2014) Controlling asthma by training of Capnometry-Assisted Hypoventilation $(\mathrm{CATCH})$ vs slow breathing: a randomized controlled trial. Chest $46(5)$ : 1237-1247.

Sep 15, 2014 - The term central sleep apnea encompasses a heterogeneous group of ... While this study failed to show a mortality benefit, CPAP was associated with ... set as a back-up rate, especially when the central apneas are long.

\section{CPAP Should Not Be Used for Central Sleep Apnea in}

www.aasmnet.org/.../020405.pdf

American Academy of Sleep Medicine

by S Javaheri - 2006 - Cited by 38 - Related articles 